\title{
REFLEXÕES SOBRE AS BASES CONCEITUAIS QUE FUNDAMENTAM A CONSTRUÇÃO DO CONHECIMENTO ACERCA DA LOMBALGIA NA GESTAÇÃO
}

Cristine Homsi Jorge Ferreira ${ }^{1}$

Ana Márcia Spanó Nakano ${ }^{2}$

Ferreira CHJ, Nakano AMS. Reflexões sobre as bases conceituais que fundamentam a construção do conhecimento acerca da lombalgia na gestação. Rev Latino-am Enfermagem 2001 maio; 9(3):95-100.

A lombalgia na gestação acomete cerca de $50 \%$ das gestantes, causando importantes transtornos sociais. Apesar disso, ela vem sendo considerada como uma ocorrência normal e até esperada na gravidez, o que tem contribuído para a falta de adoção de medidas profiláticas e de alivio. Buscamos neste trabalho compreender as bases conceituais históricas que originaram esta naturalização, usando como recorte a obstetrícia e como referência a literatura mundial sobre o tema indexada de 1987 à 1997. Apreendemos que a visão determinista relacionada ao problema respalda-se no modelo biomédico. Assim, a adoção de soluções efetivas requer a incorporação de um novo paradigma.

UNITERMOS: lombalgia, gravidez, saúde da mulher

\section{LOW BACK PAIN IN PREGNANCY IN THE PERSPECTIVE OF THE BIOMEDICAL MODEL: A CRITICAL ANALYSIS}

Low back pain is a problem that is faced by $50 \%$ of pregnant women and causes important social trouble. In spite of this, the problem is considered to be normal and is expected during pregnancy, which has contributed to the lack of prophylactic and relief measures. This work aims at understanding the historical and conceptual bases that have given rise to such naturalization as outlined by midwifery and by using world literature indexed on the topic from 1987 to 1997 as a theoretical framework. We have apprehended that a deterministic view related to this issue is supported by the biomedical model. Therefore, the adoption of effective solutions requires the incorporation of a new paradigm.

KEY WORDS: low back pain, pregnancy, women's health

\section{REFLEXIONES SOBRE LAS BASES CONCEPTUALES QUE FUNDAMENTAN LA CONSTRUCCIÓN DEL CONOCIMIENTO A CERCA DE LA LUMBALGIA EN EL EMBARAZO}

La Lumbalgia en la gestación afecta cerca de $50 \%$ de las gestantes, causando importantes transtornos sociales. Aun ésta morbilidad es considerada como normal y es esperada durante la gravidez, lo que hay contribuido para la falta de adopción de medidas profilácticas y de alivio. Buscamos en este trabajo comprender las bases conceptuales históricas que originaron ésta concepción, usando como recorte la obstetricia y como referencia la literatura mundial sobre el tema indexada de 1987 a 1997. Aprendimos que la visión determinista relacionada con el problema se respalda en el modelo biomédico. Asi, la adopción de soluciones efectivas requiere la incorporación de un nuevo paradigma.

TÉRMINOS CLAVES: lumbalgia, gestación, salud de la mujer

${ }_{1}^{1}$ Fisioterapeuta, Doutoranda do Programa de Pós-Graduação da Escola de Enfermagem da Universidade de São Paulo. E-mail: acferrei@keynet.com.br ${ }^{2}$ Enfermeira, Professor Doutor da Escola de Enfermagem de Ribeirão Preto da Universidade de São Paulo, Centro Colaborador da OMS para o desenvolvimento da pesquisa em enfermagem. E-mail: nakano@eerp.usp.br 


\section{INTRODUÇÃO}

$\boldsymbol{E}_{\mathrm{m}}$ 1899, a lombalgia era um dos problemas mais negligenciados na gestação ${ }^{(1)}$. Tal situação ainda se sustenta nos dias de hoje, sob a fundamentação de que a lombalgia é um desconforto inerente ao período gestacional e, portanto, de ocorrência normal não requerendo adoção de medidas preventivas ou de alívio ${ }^{(2-6)}$.

Em nossa prática como profissionais de saúde que trabalham com gestantes, percebemos as repercussões dessas concepções que invisibilizam o problema, verificada pela não indicação de medidas de alívio a um grande número de gestantes que se queixam de lombalgia e pelas repercussões negativas deste sintoma em suas vidas. Existem medidas preventivas à serem adotadas antes e durante a gestação, que deveriam ser difundidas por todos os que trabalham com a saúde da mulher. No entanto, percebemos que o maior empecilho para a indicação dessas medidas é o fato dos próprios profissionais de saúde não considerarem esta queixa importante.

Diante de tal problema, buscamos, neste trabalho, compreender a lógica que concebe um sintoma que traz importantes consequêencias para a mulher, apenas como uma característica normal da gestação. Acreditamos que a desconstrução dessa lógica, poderá contribuir para uma conscientização entre os profissionais de saúde, possibilitando uma nova visão não só do sintoma, mas também da mulher-gestante que sofre com ele e dos seus significados no contexto em que se insere. As reflexões que apresentamos estão fundamentadas na revisão da literatura mundial sobre o tema, indexada no período de 1987 à 1997, quando se verifica um cresceste interesse por parte dos estudiosos, relacionado à lombalgia na gestação em razão, principalmente, dos prejuízos econômicos causados por afastamentos do trabalho. Assim, em um primeiro momento faremos uma breve revisão dos principais aspectos relacionados a este tema à fim de posteriormente buscar compreender as bases conceituais que fundamentam a visão da lombalgia como característica normal da gestação, sob o recorte do corpo de conhecimento/saber da obstetrícia.

\section{LOMBALGIA NA GESTAÇÃO: PREVALÊNCIA, REPERCUSSÕES PARA A SAÚDE DA MULHER, ETIOLOGIA E MEDIDAS DE ALÍVIO}

A lombalgia é conceituada como toda condição de dor, dolorimento ou rigidez, localizada na região inferior do dorso, em uma área situada entre o último arco costal e a prega glútea. Ela, freqüentemente, é acompanhada pela lombociatalgia, que se constitui de dor que se irradia daquela região para uma ou ambas as pernas $^{(7)}$.

Vários estudos prospectivos e retrospectivos verificaram uma prevalência de lombalgia na gestação variável de $49 \%$ à $71 \%^{(2,}$ ${ }^{4,8-10)}$, com incidência de $54,8 \%^{(3)}$ e de $78 \%^{(11)}$.

Mais de um terço das mulheres grávidas se referem a lombalgia como um problema severo, que interfere em suas atividades de vida diária e capacidade de trabalho, além de contribuir para insônia por se manifestar durante a noite ${ }^{(12)}$.

Muitas mulheres se preocupam em saber como serão capazes de cuidar de um recém-nascido, estando incapacitadas pela dor $^{(13)}$. Adicionalmente, ficam muito inseguras, já que, sendo intensa a dor e estando sua causa mal definida, experimentam um forte medo de que algo errado possa estar ocorrendo com o bebê ou com a gestação, gerando, assim, intenso estresse.

As gestantes que sofrem do problema podem apresentar importante desabilitação da marcha ${ }^{(1)}$.

Além do sofrimento causado às mulheres durante o período gestacional, alguns trabalhos indicam suas conseqüências, a longo prazo, como a regressão lenta da dor no pós- parto, especialmente nas mulheres que tiveram dor de alta intensidade durante a $\operatorname{gestação}^{(9-10)}$.

Apesar de não existir um consenso sobre a etiologia da lombalgia na gestação as três hipóteses explicativas historicamente mais difundidas são da lombalgia decorrente de eventos fisiológicos próprios da gestação devido a modificações biomecânicas, hormonais e vasculares ${ }^{(1,14-16)}$. Tais hipóteses acabam corroborando a visão determinista que se estabeleceu do problema, uma vez que restringe o mesmo a uma conseqüência inevitável da fisiologia da gestação.

Parece evidente que a etiologia da lombalgia na gestação é multifatorial e que a gestação por si só pode contribuir para agravar quadros dolorosos prévios ou para o desencadeamento dos casos que se iniciam nesse período, porém a escassez de estudos sobre outros determinantes perpetua a lombalgia como evento próprio da gestação, diminuindo as possibilidades de que sejam adotadas medidas eficazes de alívio ${ }^{(17)}$.

A gestação limita a adoção das condutas diagnósticas e terapêuticas, normalmente utilizadas para a lombalgia fora da gestação, como os exames radiológicos e o uso de alguns fármacos. Assim, as medidas de alívio viáveis nesse período requerem primeiramente a valorização de ações normalmente relegadas a um segundo plano pelo modelo vigente, como a aquisição de novos hábitos posturais, a adequação dos ambientes de trabalho, a orientação ergonômica, além de exercícios terapêuticos específicos e técnicas de relaxamento ${ }^{(18)}$. 


\section{AS BASES CONCEITUAIS QUE FUNDAMENTAM A CONSTRUÇÃO DO CONHECIMENTO SOBRE A LOMBALGIA COMO CARACTERÍSTICA NORMAL DA GESTAÇÃO}

A divisão hierarquizada e especializada ainda vigente nas profissões de saúde faz com que a população em geral tenha como referência o profissional considerado "especializado" em determinada ocorrência da vida ou em determinada doença. As questões relacionadas a gestação, como a lombalgia, são consideradas de domínio da obstetrícia, que tem seu corpo de conhecimentos estruturado com base no modelo biomédico. Este modelo tem sua origem no século XVIII, através de Morgani e Bichat, com o aparecimento da anatomiapatológica, e se afirma a partir da Revolução Industrial, pelas necessidades do capitalismo, que organiza a instituição médica, estabelecendo o conceito de normalidade para atender às necessidades da produção ${ }^{(19)}$.

A clínica submete a epidemiologia e vai instrumentalizando o saber anátomopatológico, para recortar o seu objeto de trabalho e apreendê-lo, ou seja, o corpo doente e a doença, concentrandose na sua determinação biológica, minimizando outros aspectos relevantes, tais como os sociais, políticos, ambientais e culturais.

A explicação da causa da doença na biologia do indivíduo, assenta sua autoridade nos postulados de "cientificidade e neutralidade moral”(20). Neste sentido, o paradigma cientificista, estabelece os seus critérios de reconhecimento das intervenções técnicas, através de sua comprovação por modelos experimentais ${ }^{(21)}$. Em tais modelos, não cabem variáveis que ultrapassem o sujeito portador de uma patologia (anormalidade), determinadas por comparação com as funções esperadas para um organismo sadio (normal). Quando o indivíduo refere tais variáveis, as mesmas são atribuídas à ignorância, a desvios de conduta ou outros fenômenos psíquicos. Dentro desta concepção, a gestação passa a ser vista, à partir do século XVIII, como evento fisiológico mas se torna cada vez mais objeto de intervenção médica.

Algumas teorias procuraram definir a gestação não só como evento fisiológico, mas como uma relação harmônica e simbiótica entre a mãe e o feto, como na teoria da simbiose harmônica e homogênia de Bar ${ }^{(22)}$.

É bem verdade que algumas doenças maternas exemplificam claramente essa teoria, como é o caso da artrite reumatóide, que involui consideravelmente na gestação. Porém, as profundas e rápidas modificações necessárias à manutenção e desenvolvimento da gestação exigem o funcionamento de alguns órgãos maternos no limite de sua capacidade máxima.

Após a nidação do ovo, são necessárias modificações imediatas do aparelho genital. Essas transformações morfológicas, volumétricas e de consistência da genitália, proporcionarão um ambiente interno adequado para o desenvolvimento, proteção e oportuna expulsão do concepto. Em nível sistêmico, ocorrem profundas alterações cardiovasculares, pulmonares, urinárias, digestivas e músculo-esqueléticas. Paradoxalmente, as modificações locais do aparelho reprodutor que ocorrem imediatamente após a nidação, não podem ser verificadas precocemente, através de exame clínico, embora sejam predominantemente de ordem anatômica. As alterações sistêmicas, apesar de se instalarem progressivamente, no sentido de atenderem as modificações metabólicas, formação dos tecidos e a constituição de reserva para a vida neonatal, aparecem em livros tradicionais de obstetrícia como causadoras de sintomatologia materna importante, logo no início da gestação ${ }^{(22-}$ 23).

Algumas queixas, por serem muito freqüentes e por não caracterizarem doenças definidas, passam a ser consideradas como sinais característicos da gravidez e portanto parte desse processo fisiológico. Náuseas, vômitos, pirose, mericismo, sialorréia, constipação, vertigem, palpitações, dor pré-cordial, sonolência, insônia, cãibras, astenia, depressão, pruridos, leucorréia, polaciúria, varizes e a lombalgia são algumas das queixas freqüentes na gestação.

A não especificidade de tais queixas, sob o ponto de vista do modelo biomédico, passa a ser determinante para que elas sejam, de certa forma, desconsideradas.

A obstetrícia delimita o seu território de atuação, também no século XVIII quando se descobre o mecanismo da ovulação, reforçando-se à idéia de que a função da mulher, é primordialmente a de reprodutora ${ }^{(24)}$. Para o modelo biomédico que começava a imperar, a obstetrícia representa a necessária diminuição de mortalidade materna e fetal. No século XIX, o poder médico, aliado ao sistema econômico, busca assegurar a reprodução e a preservação de mão de obra para as indústrias. A obstetrícia, para dar conta de seu grande desafio, a baixa dos índices de mortalidade materna e fetal, concentra-se em doenças que possam, de alguma forma, ameaçar a gestação. A propedêutica obstétrica apreende e sistematiza essas doenças através de exames que não só as comprovem, mas também indiquem o caminho certo para o seu tratamento ${ }^{(24-25)}$. Ao mesmo tempo, a mulher se torna, cada vez mais, simples espectadora da condução de seu próprio processo gestacional. 0 seu corpo passa a ser não somente objeto de estudo pela medicina, mas também de controle e normatização, definindose o que é relevante ou não para ela ${ }^{(25)}$.

No século XX, ocorre a transferência do local de nascimento, do domicilio para o hospital, o que determina o perfil comportamental da mulher, esperado pela obstetrícia: uma boa paciente, calma, obediente, conformada, controlada, que não 
perturbe as outras nem o pessoal do serviço ${ }^{(26)}$. Essa passividade que permite o controle da mulher pela instituição, é, em muito, corroborada pela construção social da função materna como "vocação natural" da mulher, e idealização de uma maternidade de sacrifício e abnegação. Tal mito que impõe à mulher "a obrigação de ser mãe antes de tudo", foi construído no século XVIII, para atender aos interesses do estado que começava a identificar nos homens sua maior riqueza e, nas mulheres, a viabilidade para aumento e a preservação desta riqueza, através de sua responsabilização pela vida e saúde de seus filhos ${ }^{(27)}$.

A apropriação deste discurso pela medicina legitima os esteriótipos da maternidade, tendo como base a própria capacidade reprodutiva das mulheres. A maior parte das abordagens clínicas da maternidade repousam na evocação do "natural" altruísmo da mãe, sendo que o sacrifício materno é inerente à própria condição biológica da mulher.

As relações sociais de gênero se reproduzem na relação mulher/clínica médica. A imagem estereotipada da maternidade, predominante no discurso médico, revela um dos procedimentos típicos da institucionalização dos elementos centrais do sistema de sexo-gênero vigentes, pelo qual se configura o domínio masculino segundo as formas de tratar as questões de sexo, gênero e os bebês ${ }^{(28)}$. A natureza passa a funcionar como uma essência da qual podem ser apagadas diferenças sociais, historicamente instituídas, como o sexismo.

Dentro desse quadro, as queixas rotuladas como "normais" durante a gestação e, portanto, consideradas parte de um processo fisiológico, são portadoras de pouco significado, por não serem palpáveis e não representarem ameaça relevante ao desenvolvimento da gravidez. A gestante que se queixa demasiadamente desses sintomas, pode ser taxada de "poliqueixosa" porque esse tipo de desconforto é esperado e perfeitamente suportável para o período gestacional ${ }^{(5)}$.

Se a gestante sofre de lombalgia, e isto é considerado normal, logo passa a ocorrer uma "naturalização" desse tipo de dor, estabelecendo-se, assim, um determinismo em relação ao problema. Esse determinismo ancora suas explicações somente em aspectos biológicos, encobrindo outros determinantes como os sociais, políticos, culturais e psicológicos.

Sabemos que nem toda lombalgia que se manifesta no período gestacional tem como fator desencadeante a própria gestação. Aliás, grande parte das lombalgias já existiam antes da mulher engravidar, persistindo ou se agravando neste período ${ }^{(8)}$. Isto significa que a lombalgia na gestação deve ser analisada sob diversos prismas e não simplificada e banalizada em função do próprio processo gestacional.

Neste sentido, é importante a compreensão da noção de "desgaste" perpetrada como o processo biopsíquico que o ser humano desenvolve ao longo da vida como resultado das formas específicas de trabalhar e viver em sociedade ${ }^{(29)}$. Dentro dessa ótica, a lombalgia na gestação não é um processo biológico, determinado pela gravidez, mas assume formas específicas determinadas pelo modo como se dá a relação entre a mulher com a natureza e sua própria natureza: social, biológica e psicológica. Dentre esses aspectos destacam-se, os de ordem ocupacionais, que têm importante influência na gênese das lombalgias na população em geral, mas no entanto não têm merecido a devida atenção nos estudos sobre a lombalgia na gestação ${ }^{(17)}$.

Os distúrbios e doenças ocupacionais acometem, com maior freqüência, as categorias profissionais menos qualificadas, exatamente aquelas que concentram maior número de mulheres ${ }^{(29)}$. Além disso, o trabalho doméstico e cuidados com as crianças, ainda Ihes são atribuídos, contribuindo, assim, para maior desgaste físico e psíquico, devido ao acúmulo de funções.

As condições de vida e de trabalho, assim como as repercussões da dupla jornada e do estresse sobre a mulher, devem ser integradas em um quadro explicativo mais amplo acerca da lombalgia na gestação, que, ao invés de naturalizar o sofrimento, proponha soluções efetivas à partir da consideração da mulher em toda a sua complexidade humana, e não reduzida aos rótulos de reprodutora e gestante.

\section{CONSIDERAÇÕES FINAIS}

A gravidez é vista hoje como um evento fisiológico. No processo de construção histórica dessa visão utilizou-se uma classificação que levava em consideração os sinais e sintomas "mais palpáveis" da gestação que pudessem indicar alguma doença, relegando-se também à condição fisiológica e normal da gestação outros sintomas freqüentes nesse período. Apreendemos que as bases conceituais que fundamentam as concepções sobre a lombalgia na gestação encontram-se respaldadas no modelo biomédico e na obstetrícia moderna, tendo ambos sido propulsionados pelos interesses do sistema capitalista em busca de "corpos saudáveis" para a produção. Este mesmo sistema, tem o potencial de redefinir a importância das doenças e dos sintomas, conforme seus interesses. Assim, a lombalgia na gestação, nos últimos dez anos tem crescentemente merecido a atenção dos pesquisadores, que a apontam como importante causa de afastamentos do trabalho. Porém esta produção científica ainda é essencialmente sustentada pelas concepções do modelo biomédico, o qual prioriza as questões biológicas em detrimento de outros aspectos, buscando os fatores causais do problema sempre na 
própria fisiologia da gestação. Os esforços na busca de soluções na prevenção e alívio dessa morbidade, esbarram justamente na visão histórica do problema, por este ser considerado como mais um desconfor to próprio e normal da gestação. A inespecificidade dessa manifestação também tem conduzido à sua aceitação passiva, à medida que o modelo biomédico, de certa forma, dá maior "credibilidade" a queixas que fazem parte de doenças bem definidas, palpáveis e passíveis de controle. Os recursos diagnósticos e terapêuticos, normalmente utilizados para tornar mais palpável e "controlar" as lombalgias na população geral, são limitados pela própria gestação, como a utilização rotineira de radiografias e antiinflamatórios não-hormonais.

Dado o reconhecimento da necessidade de ações de alívio da lombalgia, compatíveis com o estado gestacional, há que se buscar

\section{REFERÊNCIAS BIBLIOGRÁFICAS}

1. Ostgaard HC. Assessment and treatment of low back pain in working pregnant women. Spine 1996; 20 (1):61-69.

2. Fast A, Shapiro D, Docummun EJ, Friedmann LW, Bouklas T, Floman Y. Low back pain in pregnancy. Spine 1987; 12(4):368-71.

3. Orvieto R, Achiron A, Ben-Rafael Z, Gelernter I, Achiron R. Lowback pain of pregnancy. Acta Obstet Ginecol Scand 1994; 73:20914.

4. Fung BKP, Kwong CMF, Ho ESC. Low back pain of women during pregnancy in the montainous district of central taiwan. Chinese Medical Journal 1993; 51(2):103-6.

5. Dejoseph JF, Cragin L. Biomedical and feminist perspective on low back pain during pregnancy. Nurs Clin North Am 1998; 33(4):71324.

6. Ferreira, CHJ, Nakano AS. Lombalgia na gestação: uma revisão. JBM 1999; 77(1):113-8.

7. Cecin H. Coluna cervical e lombar. In: Moreira C, Carvalho MAP. Noções Práticas de Reumatologia. Belo Horizonte (MG): Editora Health; 1996. p. 181-204.

8. Ostgaard HC, Andersson GBJ, Karlsson K. Prevalence of back pain in pregnancy. Spine 1991; 16(5):549-52.

9. Kristiansson P, Svärdsudd K, Schoultz BV. Back pain during pregnancy: a prospective study. Spine 1996; 21(6):702-9.

10. Ostgaard HC, Zetherström G, Roos-Hansson E. Back pain in relation to pregnancy: a 6 - year follow-up. Spine 1997; 22(24):294550.

11. Galão A0, Zardo EA, Paula LG. Lombalgia na gestação. Acta Médica 1995; 5:347-53.

12. Macevilly M, Buggy D. Back pain and pregnancy: a review. Pain 1996; 64:405-14. outras medidas para sua resolução. Uma vez que a percepção dessa morbidade como um problema ainda não é universal, para que medidas de prevenção e tratamento sejam adotadas é necessário que os profissionais de saúde e pesquisadores a reconheçam, dando voz às maiores interessadas, as mulheres que sofrem com ela. Porém, em geral, a formação dos profissionais de saúde ainda é fortemente dominada pelo modelo biomédico que direciona os olhares de forma reducionista e fragmentada.

A perspectiva para substituição do discurso dogmático da lombalgia, como sendo exclusivamente de natureza intrínseca à gestação, perpassa necessariamente pela incorporação de um novo olhar que leve ao desenvolvimento de competências dos profissionais de saúde em interligar propriedades biológicas ímpares à amplos aspectos do desenvolvimento humano.

13. Ostgaard HC, Andersson GBJ. Postpartum low back pain. Spine 1992; 17(1):53-5.

14. Cecin HA, Bichuetti JAN, Daguer MK, Pustrelo MN. Lombalgia e gravidez. Rev Bras Rematol 1992; 32(2):45-50.

15. Ostgaard HC, Andersson GBJ, Schultz AB, Miller JAA. Influence of some biomechanical factors on low back pain in pregnancy. Spine 1993; 18(1):61-5.

16. Heckman JD, Sassard R. Current concepts review musculoskeletal considerations in pregnancy. The Journal of bone and joint surgery 1994; 76-A (11):1720-30.

17. Ferreira CHJ. Lombalgia na gestação: paradoxo das limitações e das possibilidades de resolução do problema. [dissertação]. Ribeirão Preto (SP): Escola de Enfermagem de Ribeirão Preto/USP; 1999.

18. Polden M, Mantle J. Fisioterapia em ginecologia e obstetrícia. São Paulo (SP): Ed. Santos; 1993.

19. Gonçalves RBM. Tecnologia e organização social das práticas de saúde. São Paulo (SP): Hucitec-Abrasco; 1994.

20. Conrad P, Schneider J. Deviance and medicalization: from badness to sickness. Ohio: Columbus; 1981.

21. Grassi E, Raggio L, Montes AG. Normatização dos comportamentos reprodutivos e paradigmas médicos: estudo de caso em instituição de saúde para camadas médias. In: Costa A, Amado T. Alternativas escassas. Rio de Janeiro (RJ): Editora 34; 1994. p. 207-48.

22. Rezende J. de. A gravidez: conceito. Duração. In: Rezende J de. Obstetrícia. Rio de Janeiro (RJ): Guanabara Koogan; 1987. p.88-93. 23. Delascio D, Guariento A. Conceito de prenhez. Nomenclatura. In: Delascio D, Guariento A. Obstetrícia normal Briquet. São Paulo (SP): Savier; 1981. p. 35.

24. Tubert S. Mulheres sem sombra: maternidade e novas tecnologias reprodutivas. Rio de Janeiro (RJ): Rosa dos Tempos; 1996. 
25. Arruda A. Um atendimento ao parto para fazer ser e nascer. In: Relatório do Encontro Nacional Saúde da Mulher. Um direito a ser conquistado. Brasília; 1989.

26. Kitzinger S. Mães. Um estudo antropológico da maternidade. Lisboa: Ed. Presença; 1978.

27. Badinter E. Um amor conquistado: o mito do amor materno. $5^{a}$ ed. Rio de Janeiro: Nova Fronteira; 1985.
28. Mltjavila M, Echeveste L. Sobre a construção social do discurso médico em torno da maternidade. In: Costa A, Amado T. Alternativas escassas. Rio de Janeiro (RJ): Editora 34; 1994. p. 285-300.

29. Brenes $A C$. Conseqüências da condição feminina no processo saúde \& doença. À mulher e a saúde. Belo Horizonte: Núcleo de estudos e pesquisa sobre a mulher; 1988. 